\title{
Business information systems for the cost/energy manage- ment of water distribution networks: a critical appraisal of alternative optimization strategies
}

\author{
Candelieri A. ${ }^{1}$, Galuzzi B.G. ${ }^{1}$, Giordani I. ${ }^{1}$, Perego R. ${ }^{1}$, Archetti F. ${ }^{1,2}$ \\ ${ }^{1}$ University of Milano-Bicocca, viale Sarca 336, 20126 Milan, Italy \\ ${ }^{2}$ Consorzio Milano Ricerche, via Roberto Cozzi 53, 20125 Milan, Italy \\ antonio.candelierieunimib.it \\ bruno.galuzzi@unimib.it \\ ilaria.giordani@unimib.it \\ riccardo.perego@disco.unimib.it \\ francesco.archetti@unimib.it
}

\begin{abstract}
The objective of this paper is to show how smart water networks enable new strategies for the energy cost management of the network, more precisely Pump Scheduling Optimization. This problem is traditionally solved using mathematical programming and, more recently, nature inspired metaheuristics. The schedules obtained by these methods are typically not robust both respect to random variations in the water demand and the non-linear features of the model. The authors consider three alternative optimization strategies: (i) global optimization of black-box functions, based on a Gaussian model and the use of the hydraulic simulator (EPANET) to evaluate the objective function; (ii) Multi Stage Stochastic Programming, which models the stochastic evolution of the water demand through a scenario analysis to solve an equivalent large scale linear program; and finally (iii), Approximate Dynamic Programming, also known as Reinforcement Learning. With reference to real life experimentation, the last two strategies offer more modeling flexibility, are demand responsive and typically result in more robust solutions (i.e. pump schedules) than mathematical programming. More specifically, Approximate Dynamic Programming works on minimal modelling assumption and can effectively leverage on line data availability into robust online Pump Scheduling Optimization.
\end{abstract}

Keywords: Pump Scheduling Optimization, Bayesian Optimization, Multistage Stochastic Programming, Reinforcement Learning.

\section{Introduction}

The digital revolution in the water industry has just started, later than in other sectors notably energy, but will disrupt the sector in many, still unpredictable ways [1].

In this paper we consider the specific sector of the operations of an urban Water Distribution Network (WDN) as it is being impacted by the introduction of smart metering and the growing capability of sensing the network, specifically flow and pressures, and look to the new opportunities that this technological scenario is offering in 
the way of improving the networks operations. Indeed, the analysis of the new data streams has the potential of turning upside down all the aspects of network operations, for instance asset management, leak localization, network resilience evaluation, customer relationship management and energy cost optimization, water digital services.

Business information systems for the water sector will have to integrate these data streams and functionalities towards achieving technical and business objectives.

The authors have been active in several European and national projects, such as H2OLeak (national), ICeWater (European, FP7), DATA4WATER (European, H2020), PILGRIM (national) and PERFORM-WATER2020 (national), addressing the critical issues of leak management [2] [3], demand forecasting [4] [5] resilience evaluation [6] and, more recently energy cost optimization through innovative strategies for Pump Scheduling Optimization (PSO) [7].

Operating a WDN is a very energy intensive activity and a substantial part of this energy goes into operating pumps, either fixed or variable speed. A number of modelling and computational tools have been developed over the years [8] to optimize the energy consumption, meeting reliably customers' demand and respecting operational constraints, specifically pressure related.

PSO, described in Sec. 2, is a nonlinear problem due to the relation between flow and head pressure and its solution by mathematical programming requires some model simplification, e.g. linearization/convexification of objective functions and constraints set. Moreover, the water demand is to be assumed deterministic and known in advance. Under these conditions it's possible to apply highly efficient mixed integer linear programming (MINP) methods. Thus, the solution obtained, optimal for the model considered, might easily be unfeasible due to the simplification introduced and the assumptions on the demand. Therefore, its feasibility must be verified through a hydraulic simulation software, like EPANET[9]. Moreover, these methods require a complete and detailed knowledge of the WDN which is not always available, mostly in large scale systems built in different time stages.

Alternatively to MINP, several metaheuristics, such as Genetic Algorithms and Simulated Annealing among others, have been proposed to solve the PSO problem, such in [10], which proposes a two stage simulated annealing approach, or in [11], which uses Harmony Search to solve a multi-objective version of PSO.

The first strategy, analyzed in Sec. 3.1, is a black-box or simulation-based Global Optimization. This approach is based on Bayesian Optimization (BO) [12], does not require simplification/approximation, uses EPANET to evaluate the objective function, and to take care that the pressure constraints are satisfied.

The second strategy, reported in Sec. 3.2, is focused on the uncertainty on the water demand, whose probability distribution is assumed to be known a-priori. The aim is to approach PSO via Multi Stage Stochastic Programming (MSSP), by solving several water demand scenarios generated according to the distributions and represented through a scenario tree [13].

BO and MSSP are characterized by an off-line analysis, where the solution is the state of each pump for every time step (typically 1 hour) of the optimization horizon (typically 24 hours). However, the availability of on-line consumptions and operational data enables to formulate PSO as an on-line optimization/control problem. Indeed, the 
authors believe that the digitalization of WDN will offer opportunities for alternative optimization strategies free from the limitations of BO and MSSP and able to leverage the availability in smart grid of online data into strategies with the potential of simultaneously learning and optimizing. The third strategy, described in Sec. 3.3, is based on a Markov Decision Process (MDP), structured in term of states, transition dynamics and actions specified for each state and rewards. In this case the PSO problem is solved using the Approximate Dynamic Programming (ADP), a.k.a. Reinforcement Learning (RL) in the machine learning community, that does not require full knowledge of the system (i.e. transition dynamics and rewards), and tries actions, in different states, with the aim to learn something more about the system behavior (exploration) while exploiting current knowledge to infer a policy, even if not the best, with a good potential towards long term reward.

The three strategies can be tested both on benchmarks and real WDNs. However, since WDNs evolve along different trajectories both in terms of data model and availability as well as optimization goals, we do not provide a comparison between the numerical results obtained by using the three strategies on three different experimental settings. The main result of this paper is a set of new generation optimization strategies which, not necessarily as computationally efficient as deterministic mathematical programming, adapt to different operational and technological settings, and blend effectively learning from the environment (exploration) and optimization depending on the info gathered so far (exploitation).

Finally, in Sec. 4 we report the main conclusions and discussion about the benefits and limitations of the three strategies, depending on the different operational settings.

\section{The pump scheduling optimization problem}

A complex WDN can be represented by a set of nodes connected by links. The nodes consist of three subsets: reservoirs, tanks and junctions. Reservoirs are nodes that represent an infinite external source or sink of water to the network. Tanks are nodes with storage capacity, where the volume of water can vary with time during a simulation. Junctions are points in the network where links join and where water can leave the network to satisfy a certain quantity of water demand. We indicate with $J, R$, and $K$ the set of junctions, reservoirs, and tanks, respectively.

The links consist of two subsets: pipes, that convey water from one point in the network to another, and pumps, that are used to increase the pressure within the network by generating enough water flow to satisfy the demand. We indicate with $N$, and $N_{p}$, the set of pipes and pumps, respectively.

Different decision variables can be defined related to the WDN analyzed over a planning horizon, generally divided into equally-sized time periods $t \in\{1, \ldots, T\}$ : the status $s_{i, t}$ of the pumps $i \in N_{p}$, the flow rate $q_{i, t}$ and the head-loss $h_{i, t}$ of the pipes $i \in N$, and the volume of water $v_{k, t}$ of the tanks $k \in K$. Typical parameters of the optimization problem are the set of the demands $D_{j, t} \geq 0$, for each junction node $j \in J$.

An example of benchmark WDN very used is the Anytown [11], reported in Fig. 1a. It consists of 37 pipes, 19 nodes, 1 tank and 1 reservoir, with 4 pumps installed at the 
source of supply water. The total water demand $D_{t}=\sum_{j \in J} D_{j, t}$ is reported in Fig. $1 \mathrm{~b}$ and ranges from a minimum of 161.51 lps to a maximum of $484.53 \mathrm{lps}$, with two peaks during a 24-hour period, one around 10:00 and another around 20:00.
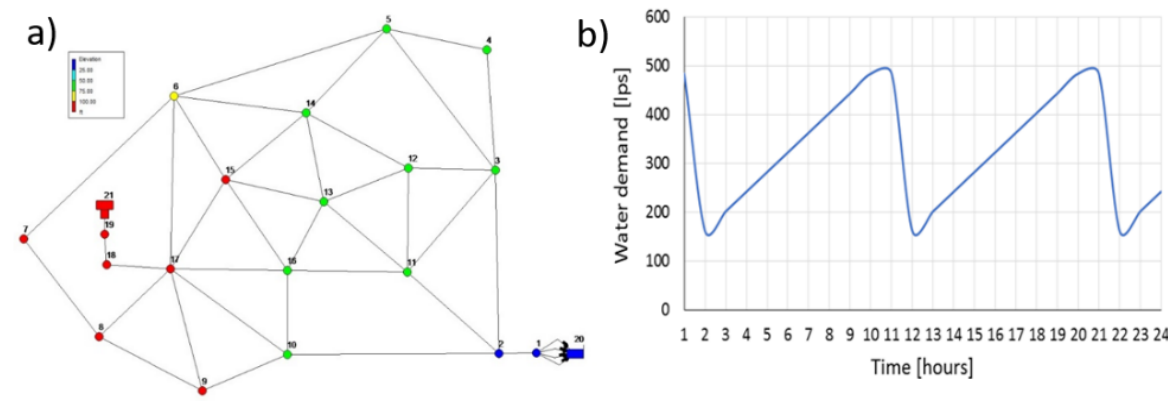

Fig. 1. a) Topology of the water distribution system Anytown. b) The hourly water demand of Anytown network over 24 hours.

Generally, the PSO problem is formulated as the minimization of an objective function, representing the value of the energy consumption on the planning horizon [8][14]:

$$
\min E,=\min \left(\sum_{i \in N_{p}} \sum_{t=1}^{T} \frac{c_{t}}{e_{i}} \cdot \lambda \cdot s_{i, t} \cdot h_{i, t} \cdot q_{i, t}\right),
$$

where $e_{i} \in(0,1)$ represents the efficiency of the pump $i \in N_{p}, C_{t}$ is the electricity cost, measured in $\$ / \mathrm{kWh}$, and $\lambda$ is an appropriate constant factor.

For feasibility reasons, the operational constraints [15] must be added to the formulation, such as the flow-rate or the water levels of tank(s) within a given range. An important set of constrains is related to flow-continuity equations for all the WDN's junctions:

$$
\sum_{i \in N_{j}} q_{i, t}=D_{j, t} \forall j \in J, \forall t \in T
$$

where $N_{j}$ indicates the subset of links with a vertex in junction $j$ and the flow $q_{i, t}$ is positive by convention. Another important set of constrains is related to the flow-headloss equations:

$$
h_{i, t}=\left\{\begin{array}{r}
h_{0}-r \cdot q_{i, t}{ }^{n}, \quad \forall i \in N_{p} \\
r \cdot p_{i, t}{ }^{n}+m \cdot q_{i, t}{ }^{2}, \forall i \in N
\end{array}, \forall t \in T\right.
$$

where $r$ is the resistance coefficient, $n$ is the flow exponent, $m$ is the minor loss coefficient, and $h_{0}$ is the shutoff head for the pump. Finally, an important constrain is the tank mass balance equation, $\forall t \in T$ :

$$
v_{k, t}=v_{k, t-1}+\sum_{i \in N_{k}} q_{i, t}, \forall k \in K,
$$

where $N_{k}$ indicates the subset of links with a vertex in the tank node $k$. 
This formulation of the PSO is a mixed integer non-linear problem that is computationally expensive to solve using a standard optimization software with no guarantee of global optimality.

The optimal pump schedule obtained by solving the PSO is limited to the decision variables $s_{i, t}$ which represent the status of each pump $i$ at time step $t$. These variables are discrete (i.e., $\{0,1\})$ in the case of on/off pumps, and continuous (e.g. in $[0,1])$ in the case of variable speed pumps. However, a simple WDN such as Anytown have 4 ON/OFF pumps and, considering an hourly resolution and a 24-hours horizon, this leads to an optimization problem with 96 discrete decision variables and, consequently, $4^{24}\left(\right.$ i.e. $\approx 2.8 \cdot 10^{14}$ ) possible pump schedules.

\section{Optimization strategies}

\subsection{Global Optimization through Bayesian Optimization}

The first strategy presented in this work to solve the PSO problem uses a Global Optimization (GO) approach and, specifically, Bayesian Optimization (BO) [16] [17]. In this case the PSO problem is considered as a solution $x^{*}$ of a global optimization problem of a black-box usually expensive-to-evaluate, objective function $f(x)$,

$$
x^{*}=\underset{x}{\operatorname{argmin}} f(x)
$$

where $x \in X \subset R^{d}$ is a point in a d-dimensional bounded-box space $X$. With respect to the PSO problem, $f(x)$ represents the energy cost $E$ (Eq. 1), whereas the dimension $d$ of the search space is given by $T \cdot N_{p}$ [7]. Each evaluation will require to run an EPANET simulation and, in case of possible warnings during the simulation run (i.e. some constraint is not satisfied) is possible to use a penalty on the objective function.

In BO the objective function $f$ is modelled as a realization of a stochastic process, typically, a Gaussian Process (GP) on a probability space $(\Omega, \Sigma, P)$. A GP, which defines a prior distribution over the function $f$, is completely specified by its mean $\mu(x): X \rightarrow R$ and a definite positive covariance function $k\left(x, x^{\prime}\right): X^{2} \rightarrow R$ :

$$
f(x) \approx G P\left(\mu(x) ; k\left(x, x^{\prime}\right)\right) .
$$

It can intuitively think of GP as analogous to a function, but instead of returning a single numeric value $f(x)$, it returns the mean and variance of a normal distribution over the possible values of $f(x)$ (Fig. 2a).

The BO algorithm starts with an initial set of $k$ points $\left\{x_{i}\right\}_{i=1}^{k} \in X$ and the associated observations $\left\{y_{i}\right\}_{i=1}^{k}$ with $y_{i}=f\left(x_{i}\right)$. At each iteration $\bar{k} \in\{\mathrm{k}+1, \ldots, \mathrm{N}\}$, the GP prior is updated using the Bayes rule, to obtain posterior distribution conditioned on the current training set $S_{t}=\left\{\left(x_{i}, y_{i}\right)\right\}_{i=1}^{\bar{k}}$ containing the past evaluated points and observations. For any point $x \in X$, the posterior mean $\bar{\mu}_{t}(x)$ and the posterior variance $\bar{\sigma}_{t}^{2}(x)$ of the GP, conditioned on $S_{t}$, are known in closed-form:

$$
x_{\bar{k}+1}=\underset{x \in X}{\arg \max } U_{\bar{k}}\left(x ; S_{\bar{k}}\right),
$$


where $U_{\bar{k}}$ is a acquisition function to maximize. Solving this auxiliary problem does not involve the evaluation of the expensive objective function $f$, but only the posterior quantities of the GP and, thus, is considered cheap. Several acquisition functions have been proposed, such as the Probability of Improvement [18], the Expected Improvement [12], the Lower Confidence Bound (LCB) and Upper Confidence Bound (UCB) [19], and the Knowledge Gradient [20].

\subsection{Multi-Stage Stochastic Programming}

The second strategy presented in this work to solve the PSO problem simplifies the original problem to a simple linear problem (LP), where the nonlinear relation coming from the objective function and/or some of the constrains above are circumvented.

A complex WDN can be simplified to only three nodes, one reservoir, one tank, and one junction, and two links, one pump from the reservoir to the junction node, and one pipe from the junction node to the tank. For each period $t$, a tank volume $v_{t}$ and a pump flow rate $q_{t}$ is defined, whereas the junction node has as demand $D_{t}=\sum_{j \in J} D_{j, t}$.

By this way, the original PSO problem is approximated by an LP problem [21] [11]:

$$
\min _{q_{1}, \ldots, q_{T}} \sum_{t=1}^{T} c_{t} \cdot q_{t},
$$

subjected to the following constrain:

$$
v^{\min } \leq v_{1}+\sum_{l=1}^{t}\left(q_{l}-D_{l}\right) \leq v^{\max }, \forall t \in T .
$$

where $c_{t}$ is an appropriate factor, representing the proportional factors between the energy and the pumped flow, and $v^{\min }$ and $v^{\max }$ represent the minimum and the maximum water level of the tank.

Such simple model can be used to describe a more complex model of water distribution in a synthesis stage [21], whereas an extended period simulation, using EPANET, can be used subsequently in order to verify the feasibility of the obtained pump schedule, in an analysis stage. If the demand pattern $\left\{D_{t}\right\}_{t=1}^{T}$ is known in advance, the optimal pumping policy is given by the optimal solution to the LP problem, solved by using any commercial code able to solve a MIL problem such as CPLEX.

The advantage of such formulation consists of the possibility to use the Multi-stage stochastic programming (MSSP) approach [22] [23], to manage a possible uncertain demand, in which, for example, the demand at each time follows a probability distribution. In this case the evolution pattern of the demand is treated as a stochastic process $\boldsymbol{D}=\left(D_{1}, \ldots, D_{T}\right)$ (sequence of random variables representing a random time series), and the flow rate $q_{t}$ as a decision process $\boldsymbol{q}=\left(q_{1}, \ldots, q_{T}\right)$, that are interlinked into a sequence of alternating decisions and observations: $q_{1}, D_{1}, q_{2}, D_{2} \ldots, q_{T}, D_{T}$. The decisions at each stage are made while considering that will be opportunities for modification and corrections at later stages (recourse decisions). The decision process is non-anticipative or implementable, i.e. the decision $q_{t}$ at time $t$ depends only on previous information $D_{1}, \ldots, D_{t-1}$. The MSSP formulation for the problem of Eq. (7) will be set as follow: 


$$
\min _{q_{1}, \ldots, q_{T}} \mathbb{E}\left[\sum_{t=1}^{T} c_{t} \cdot q_{t}\left(D_{[t]}\right)\right],
$$

subject to constraint (9) and where $q_{t}\left(D_{[t]}\right)$ means that the solution $q_{t}$ will be depends only on the demand until stage $t$ and $\mathbb{E}$ represents the expected value as a function of the demand. The MSSP solves for an optimal policy which contains the first-stage decisions (values) and the recourse decisions (functions of revealed information).

Finding the functions $q_{t}(\cdot)$ which lead to optimal solution of the MSSP is done introducing a discrete approximation of space of the stochastic process by means of the scenarios [13], which are particular possibilities of how the process might be realized as the future unfolds. The stochastic process $\boldsymbol{D}$ is discretized into a finite set of scenarios $\boldsymbol{D}^{s}=\left(D_{1}^{S}, \ldots, D_{t}^{S}\right) \in \Omega_{T}, \forall s=1, \ldots S$, with a probability $p_{s}$ where $\sum_{s=1}^{S} p_{s}=1$. Each scenario, $\boldsymbol{D}^{s}$, is defined as a possible realization of the stochastic process over the entire time horizon. The function $q_{t}(\cdot)$ is replaced by a corresponding finite number of vectors, equal to the different possibilities taken by the function $q_{t}(\cdot)$ as its input, and using a non-anticipativity requirement, that means that every pair of scenarios $\boldsymbol{D}^{k}, \boldsymbol{D}^{l} \in$ $\Omega$, which are indistinguishable up to stage $t$ (share the same history) have the same decision history up to stage $t$. A common way to represent such situation is through a scenario tree (Fig. 2) [13], consisting of nodes and arcs; each node represents a possible realization of the stochastic process, where the root node represents the first stage. In such a scenario tree, the path from the root node of the tree to each of its leaf nodes corresponds to a single scenario, and the event outcomes for scenarios that pass through the same intermediate node are identical for all stages up to that node.

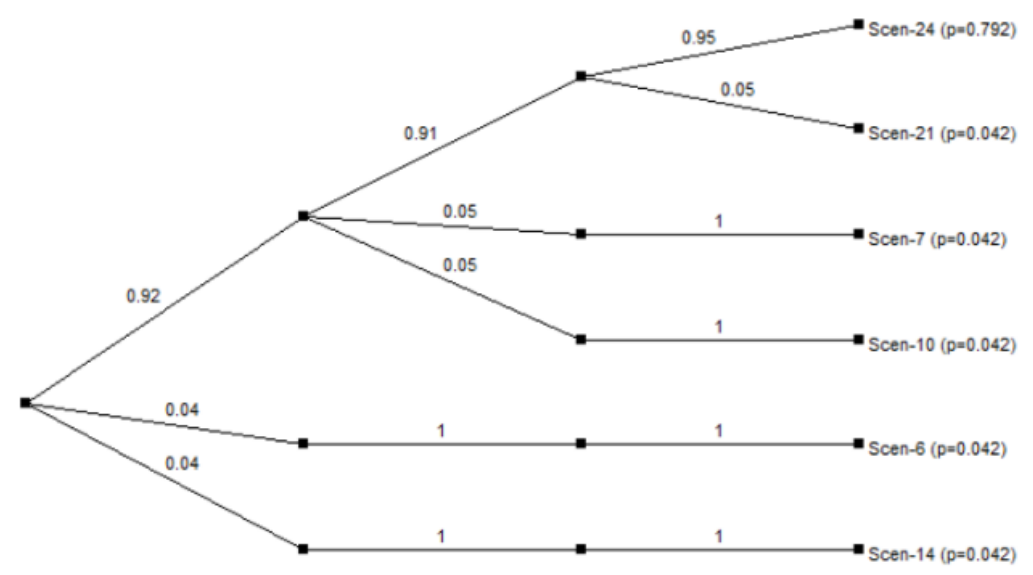

Fig. 2 Example of scenario tree.

\subsection{Approximated dynamic programming}

The third strategy, presented in this section, is based on Markov Decision Processes (MDP), a powerful framework to model a variety of sequential optimization problems and provide a robust mechanism to generate solutions online. 
An MDP is based on a state space $S$, and an action space $\mathcal{A}$. A state consists of a set of possible decision variables $s_{t}=\left\{\xi_{1, t}, \ldots, \xi_{N_{v}, t}\right\}$ at a time step $t$. An action $a_{t}=$ $a$ allows for moving from the current state $s_{t}=s$ to another state $s_{t+1}=\bar{s}$, according to the transition dynamics of the system. When transition dynamics is not known apriori, as well as the immediate rewards associated to every state-action pair, Approximate Dynamic Programming (ADP) is used. Any ADP algorithm learns how to behave with the system to be optimized by directly interacting with it (aka "environment") while infers an optimal policy (i.e. a mapping from every state $s$ to the action $a$ associated to the highest long-term reward). More precisely, we consider Q-Learning, one of the most widely adopted ADP algorithm, well-known in the Reinforcement Learning community. It takes its name from the variable $Q(s, a)$ which represents the value of being in the state $s \in S$ and taking the action $a \in \mathcal{A}$ (i.e. state-action value function).

Q-Learning works "going forward in time", where the next action to perform is selected according to an $\varepsilon$-greedy policy, selecting a random action with probability $\varepsilon$ (exploration)and the action with maximum value of $Q(s, a)$, (exploitation)with probability $1-\varepsilon$.

After the selected action is performed, an immediate reward $r$ value is observed along with the new state $s^{\prime}$, so the value of the state-action pair, $Q(s, a)$, is updated consequently:

$$
Q(s, x) \leftarrow Q(s, x)+\alpha\left[\mathcal{R}(s, x)+\gamma \max _{x^{\prime}} Q\left(s^{\prime}, x^{\prime}\right)-Q(s, x)\right]
$$

where $\alpha$ is the learning rate - which sets how much the old estimate of the Q-value has to change depending on the observed state and reward - and $\gamma$ is the discount factor - which sets how much the future rewards impact on the update of the Q-value of the current state-action pair.

In case of the PSO problem, we have defined the state as a $2 \mathrm{D}$ vector consisting of the tank level and the average pressure (computed on all the WDN's junctions). The two vector components have been discretized on 5 levels each, leading to a discrete state space with size 25 . An action is a vector with a number of components equal to the number of pumps, that is 4 in the Anytown case study. In our previous analysis we have considered on/off pumps, only [24]. Finally, the transition dynamics is not modelled - indeed, Q-Learning is "model-free" - and the reward is computed according to the energy costs associated to the actions performed.

Therefore Q-Learning does not provide, in one shot, an entire schedule for the 24 hours horizon, but suggests the best action to perform (i.e. activation of each pump) at every decision step, working online with the WDN. Q-Learning proved to be an effective strategy even when the optimal policy is obtained on a deterministic case and then applied on the real-world system: it is robust with respect to demand variations/uncertainty 


\section{Conclusion and discussion}

In this paper, we have presented several approaches because PSO in WDN should be modelled and solved in different ways according to the objectives of the analysis, the data infrastructure and availability and in general the state of digitalization of the network.

$\mathrm{BO}$ has the advantage that the problem formulation is not analytical but based on a hydraulic simulator that can take care, also in a detailed way, of operational objective and constraints. This simulation, based black-box optimization, is expensive because each evaluation of the model requires the run of simulation but has the advantage that the solutions proposed are hydraulically feasible. Moreover, BO alternates exploration stages, to acquire information on the model, and exploitation to improve the incumbent solution: this affects also early stage solutions proposed by the algorithm correspond to good quality schedules. This make possible to deal with WDN, as shown in [7], way larger than the theoretical scaling properties would suggest.

The MSSP solution we presented is analytically based and requires linearization of objective functions and constraints. This simplification makes it necessary to check the hydraulic feasibility of the proposed schedules. An advantage of the MSSP approach is that demand is no longer considered deterministic but can be uncertain, modeled through a probability distribution. The strategy is computationally demanding requiring the generation of a scenario tree and consequently a larger set of variables to simulate the possible evolution of the water demand.

The result of MSSP is not just a schedule but more generally a strategy mapping the water demand observed so far to the best flow provided by pumps for the current step (i.e. node of the scenarios tree).

The last strategy ADP/RL is completely data-driven which makes it, at least in principle, the natural choice where full digital infrastructure is already in place and can acquire and process online pressure, flows, water demand and energy consumption. There is no need to model or know a priori the water demand whose values are provided by sensor readings. Simulation is not needed because the sensory infrastructure is such that the state of the system is observable on line This strategy is also structure in phases of exploration (learning something more about the WDN behavior) and exploitation (using the current knowledge to improve on the best pump schedule obtained so far. Similarly, to MSSP, ADO/RL provides not only a solution but more generally a strategy mapping the water demand observed so far to the best flow provided by pumps for the current step (i.e. node of the scenarios tree). The difference is that in ADP/RL this strategy is inferred by interacting online with the WDN, instead of generating possible scenarios a-priori

The focus of this paper is only energy operations management strategy which is enabled by smart water networks: besides the PSO problem it is important to remark that other operational functions are going to be disrupted by the online data availability as already demonstrated by the prototypes developed by the authors for leak management [25], demand forecasting [26] [27] and resilience evaluation [28] [29] in the previously cited European projects. 


\section{References}

[1] R. A. Stewart et al., "Integrated intelligent water-energy metering systems and informatics: Visioning a digital multi-utility service provider," Environ. Model. Softw., vol. 105, pp. 94-117, 2018.

[2] A. Candelieri, I. Giordani, and F. Archetti, Automatic configuration of kernel-based clustering: an optimization approach, vol. 10556 LNCS. 2017.

[3] A. Candelieri, D. Soldi, and F. Archetti, "Cost-effective sensors placement and leak localization - The Neptun pilot of the ICeWater project," J. Water Supply Res. Technol. - AQUA, vol. 64, no. 5, pp. 567-582, 2015.

[4] S. Shabani, A. Candelieri, F. Archetti, and G. Naser, "Gene expression programming coupled with unsupervised learning: A two-stage learning process in multi-scale, shorttermwater demand forecasts," Water (Switzerland), vol. 10, no. 2, 2018.

[5] A. Candelieri et al., "Tuning hyperparameters of a SVM-based water demand forecasting system through parallel global optimization," Comput. Oper. Res., 2018.

[6] A. Candelieri, I. Giordani, and F. Archetti, "Supporting Resilience Management of Water Distribution Networks through Network Analysis and Hydraulic Simulation," in Proceedings - 2017 21 st International Conference on Control Systems and Computer, CSCS 2017, 2017, pp. 599-605.

[7] A. Candelieri, R. Perego, and F. Archetti, "Bayesian optimization of pump operations in water distribution systems," Springer US, 2018.

[8] H. Mala-Jetmarova, N. Sultanova, and D. Savic, "Lost in Optimisation of Water Distribution Systems? A Literature Review of System Design," Water, vol. 10, no. 3, p. 307, 2018.

[9] L. a Rossman, "EPANET 2: users manual," Washington, DC, 2000.

[10] G. McCormick and R. S. Powell, "Derivation of near-optimal pump schedules for water distribution by simulated annealing," J. Oper. Res. Soc., vol. 55, no. 7, pp. 728-736, 2004.

[11] F. De Paola, N. Fontana, M. Giugni, G. Marini, and F. Pugliese, "An Application of the Harmony-Search Multi-Objective (HSMO) Optimization Algorithm for the Solution of Pump Scheduling Problem," Procedia Eng., vol. 162, pp. 494-502, 2016.

[12] J. Mockus, Bayesian Approach to Global Optimization. Springer Netherlands, 1989.

[13] J. Dupacová, G. Consigli, and S. W. Wallace, "Scenarios for Multistage Stochastic Programs," Ann. Oper. Res., vol. 100, no. 1, pp. 25-53, 2000.

[14] B. Ghaddar, J. Naoum-Sawaya, A. Kishimoto, N. Taheri, and B. Eck, "A Lagrangian decomposition approach for the pump scheduling problem in water networks," Eur. J. Oper. Res., vol. 241, no. 2, pp. 490-501, 2015.

[15] C. D'Ambrosio, A. Lodi, S. Wiese, and C. Bragalli, "Mathematical programming techniques in water network optimization," Eur. J. Oper. Res., vol. 243, no. 3, pp. 774$788,2015$.

[16] J. Močkus, "On bayesian methods for seeking the extremum," Lect. Notes Comput. Sci. (including Subser. Lect. Notes Artif. Intell. Lect. Notes Bioinformatics), vol. 27 LNCS, pp. 400-404, 1975.

[17] F. Archetti and B. Betrò, "A probabilistic algorithm for global optimization," Calcolo, vol. 16, no. 3, pp. 335-343, 1979. 
[18] H. J. Kushner, "A new method of locating the maximum point of an arbitrary multipeak curve in the presence of noise," J. Basic Eng., vol. 86, no. 1, pp. 97-106, 1964.

[19] Auer, "Using Confidence bounds for Exploration Exploitation trade-offs," Jmlr, vol. 3, pp. 397-422, 2002.

[20] P. I. Frazier, "Knowledge-Gradient Methods for Statistical Learning," Princeton University, 2009.

[21] A. P. Goryashko and A. S. Nemirovski, "Robust energy cost optimization of water distribution system with uncertain demand," Autom. Remote Control, vol. 75, no. 10, pp. 1754-1769, 2014.

[22] V. Puleo, M. Morley, G. Freni, and D. Savić, "Multi-stage linear programming optimization for pump scheduling," Procedia Eng., vol. 70, pp. 1378-1385, 2014.

[23] M. Housh, A. Ostfeld, and U. Shamir, "Limited multi-stage stochastic programming for managing water supply systems," Environ. Model. Softw., vol. 41, pp. 53-64, 2013.

[24] Candelieri A, R. Perego, and F. Archetti, "Intelligent Pump Scheduling Optimization in Water Distribution Networks," in 12th International Conference, LION 12, Kalamata, Greeece., 2018.

[25] A. Candelieri, F. Archetti, and E. Messina, "Analytics for supporting urban water management," Environ. Eng. Manag. J., vol. 12, no. 5, pp. 875-881, 2013.

[26] A. Candelieri, "Clustering and support vector regression for water demand forecasting and anomaly detection," Water (Switzerland), vol. 9, no. 3, 2017.

[27] A. Candelieri, D. Soldi, and F. Archetti, "Short-term forecasting of hourly water consumption by using automatic metering readers data," in Procedia Engineering, 2015, vol. 119, no. 1, pp. 844-853.

[28] A. Candelieri, D. Soldi, and F. Archetti, "Network analysis for resilience evaluation in water distribution networks," Environ. Eng. Manag. J., vol. 14, no. 6, pp. 1261-1270, 2015.

[29] D. Soldi, A. Candelieri, and F. Archetti, "Resilience and vulnerability in urban water distribution networks through network theory and hydraulic simulation," Procedia Eng., vol. 119, no. 1, pp. 1259-1268, 2015. 